\title{
Measurement Uncertainty Impacts Diagnosis of Diabetes Mellitus: Reliable Minimal Difference of Plasma Glucose Results
}

\author{
Sandra Keutmann · Stephanie Zylla • Mathilde Dahl • Nele Friedrich • \\ Rüdiger Landgraf · Lutz Heinemann · Anders Kallner • \\ Matthias Nauck · Astrid Petersmann
}

Received: August 22, 2019 / Published online: December 16, 2019

(c) The Author(s) 2019

\begin{abstract}
Introduction: The diagnosis of diabetes mellitus is based on suitable cut-off values of specific biomarkers, such as the concentration of glucose in plasma. The German Diabetes Association has very recently published a clinical practice guideline on the definition, classification and diagnosis of diabetes mellitus that recommends measurements of plasma glucose concentration have an imprecision defined as a minimal difference (MD) of at a fasting plasma
\end{abstract}

Enhanced Digital Features To view enhanced digital features for this article go to https://doi.org/10.6084/ m9.figshare.10790468.

Electronic supplementary material The online version of this article (https://doi.org/10.1007/s13300019-00740-w) contains supplementary material, which is available to authorized users.

S. Keutmann · S. Zylla · M. Dahl - N. Friedrich . M. Nauck · A. Petersmann ( $\square)$ Institute of Clinical Chemistry and Laboratory Medicine, University Medicine Greifswald, Greifswald, Germany

e-mail: astrid.petersmann@med.uni-greifswald.de

S. Zylla $\cdot$ N. Friedrich $\cdot$ M. Nauck

DZHK (German Centre for Cardiovascular Research), Partner Site Greifswald, University

Medicine Greifswald, Greifswald, Germany

R. Landgraf

German Diabetes Foundation (DDS), Munich, Germany glucose concentration of $7.0 \mathrm{mmol} / \mathrm{L}$. To obtain reliable values for the MD, we investigated longterm and short-term measurement uncertainty. Methods: The imprecision was determined by two approaches: (1) a long-term dataset with imprecision based on the Guideline of the German Medical Association on Quality Assurance in Medical Laboratory Examinations (RiliBAEK), in a medical laboratory operating $24 / 7$, using internal quality control (IQC) data for four concentrations during a 10-year period; and (2) a detailed short-term dataset with imprecision assessed by hourly measurements of control materials. These datasets were used to calculate the MD cut-off ( $\left.\mathrm{MD}_{\text {cut-off }}\right)$ as: $\mathrm{MD}_{\text {cut-off }}=k \times \mathrm{SD}$ $=2 \times \mathrm{SD}$, where SD is the standard deviation and $k=2$ represents a confidence level of $95 \%$.

Results: The $\mathrm{MD}_{\text {cut-off }}$ of $\leq 0.7 \mathrm{mmol} / \mathrm{L}$ at a fasting plasma glucose concentration of $7.0 \mathrm{mmol} / \mathrm{L}\left(\mathrm{MD}_{\text {cut-off }} 7.0\right)$ for the long-term and

\section{Heinemann}

Science-Consulting in Diabetes $\mathrm{GmbH}$, Neuss, Germany

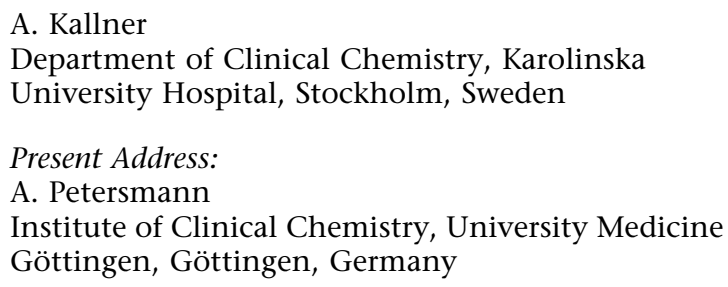

Present Address:

A. Petersmann

Institute of Clinical Chemistry, University Medicine Göttingen, Göttingen, Germany 
the short-term approaches were 0.44 and $0.40 \mathrm{mmol} / \mathrm{L}$, respectively. The $\mathrm{MD}_{\text {cut-off } 7.0}$ from both approaches was therefore below the recommended value of $0.7 \mathrm{mmol} / \mathrm{L}$. It was noted that the variability in performance within and between instruments can be covered by reporting the long-term $\mathrm{MD}_{\text {cut-off } 7.0}$ across all connected instruments. In this study, stable results for the $\mathrm{MD}_{\text {cut-off } 7.0}$ were obtained after 1 year.

Conclusion: Imprecision as measured by IQC data is remarkably stable over many years of operation. Current imprecision assessment usually focuses on only single instruments, whereas clinicians perceive the measurement as the result of the combined analytical performance of all instruments used for a certain assay. In the clinical setting, the MD may be a more useful measure of imprecision, and we suggest deriving the $\mathrm{MD}_{\text {cut-off }}$ combined from all instruments and control cycles that are used in the patient care setting for a given analyte.

Keywords: Assay performance; Coefficient of variation; Imprecision; Internal quality control; Minimal difference; Rili-BAEK; Westgard rules

\section{Key Summary Points}

All laboratory measurements are subject to measurement uncertainty, which can be described using the coefficient of variation $(\% \mathrm{CV})$. Despite the pervasive presence of high measurement uncertainty and the enormous impact it may have on the diagnosis of diabetes mellitus (DM), measurement uncertainty has not received appropriate attention by clinical users.

Our aim was to create awareness of the fact that the magnitude of measurement uncertainty especially impacts the diagnosis of DM when cut-off values are used. We calculated the recommended "minimal difference (MD)", a value recently developed for this purpose, based on detailed short-term and long-term (10 years) internal quality control data for state-of-the-art glucose concentration measurements.
The MD represents the smallest difference between two values that is statistically significant, taking the specific measurement uncertainty into account; it is based on the standard deviation and expressed in the unit of the measurand to enable easy use by clinicians.

At the fasting plasma glucose cut-off of $7.0 \mathrm{mmol} / \mathrm{L}(126 \mathrm{mg} / \mathrm{dL})$, which represents a frequently used cut-off value for the diagnosis of DM, the MD cut-off $\left(\mathrm{MD}_{\text {cut-off }} 7.0\right)$ for the investigated assay was $0.44 \mathrm{mmol} / \mathrm{L}$ and remained below the recommended maximum MD of $0.7 \mathrm{mmol} / \mathrm{L}$.

State-of-the-art glucose concentration measurements with low imprecision are associated with a considerable MD and therefore may impact cut-off points for diagnosing diabetes. It is important to note that imprecisions allowed for by guidelines or legal requirements are considerably higher than those observed in this study. Consequently, imprecision in glucose assays should also be reported as MD to enable clinical users to consider imprecision when relying on the results of a glucose assay to diagnose DM.

\section{INTRODUCTION}

The diagnosis of diabetes mellitus (DM) is based on suitable cut-off values for specific biomarkers, such as the plasma glucose concentration. The World Health Organization (WHO), American Diabetes Association (ADA) and German Diabetes Association (DDG) recommend assaying samples of venous plasma, with a cut-off for fasting plasma glucose (FPG) of $\geq 7.0 \mathrm{mmol} / \mathrm{L}$ ( $\geq 126 \mathrm{mg} / \mathrm{dL}$ ) and for 2-h plasma glucose of $\geq 11.1 \mathrm{mmol} / \mathrm{L}(200 \mathrm{mg} / \mathrm{dL})$ after ingestion of $75 \mathrm{~g}$ of glucose in the oral glucose tolerance test [1-3]. According to WHO, impaired fasting glucose (IFG) is diagnosed when the plasma glucose 
level is between 6.1 and $6.9 \mathrm{mmol} / \mathrm{L}(110-125$ $\mathrm{mg} / \mathrm{dL}$ ); according to ADA and DDG, the interval in plasma glucose level for IFG diagnosis is $5.6-6.9 \mathrm{mmol} / \mathrm{L}(100-125 \mathrm{mg} / \mathrm{dL})$ [3].

\section{A Laboratory Result is Complete only when Reported Together with the Associated Measurement Uncertainty}

Laboratory reports are often treated like a bank account statement: the value that is given on the statement is understood to be the actual amount of money in the bank account, and deviation by a few cents more or less is not acceptable. In contrast to the values on bank account statements, however, laboratory measurements are always subject to measurement uncertainty, and a few "cents" more or less in the results may represent the same value, depending on the magnitude of the associated uncertainty. Although it is generally accepted that a measurement result is only complete if the associated uncertainty is reported together with the measurement result, as stated in "The Guide to the Expression of Uncertainty in Measurements" [4], the uncertainty is usually not reported to the clinical end-user. Medical laboratories commonly report imprecision as the coefficient of variation $(\% \mathrm{CV})[5]$. The $\% \mathrm{CV}$, as given in Eq. (1), may be difficult to immediately translate to a difference between two consecutive results or between a result and a cut-off value. It is important that the end-userthe clinician-takes the measurement uncertainty into account when comparing a laboratory result to a recommended cut-off value, such as, for example, when diagnosing or treating DM. Therefore, the uncertainty expressed in the units of the measurand, i.e. the minimal difference (MD), would be a more useful metric.

\section{Description of Minimal Difference}

The DDG recently published a clinical practice guideline on the definition, classification and diagnosis of DM that recommends minimum requirements for the imprecision of glucose concentration measurements, expressed as the MD [2]. The MD is not a novel metric and has been described previously-for example, in the approved guideline of the Clinical and Laboratory Standards Institute (CLSI) EP29-A on "Expression of Measurement Uncertainty in Laboratory Medicine" [6]. The MD represents the smallest difference between two values that is statistically significant, taking the specific measurement uncertainty into account. It is based on the standard deviation (SD) and is expressed in the unit of the measurand so that clinicians are able to apply it directly to the result [7].

At the FPG cut-off of $7.0 \mathrm{mmol} / \mathrm{L}$, the DDG recommends a $\mathrm{MD}$ cut-off $\left(\mathrm{MD}_{\text {cut-off }}\right)$ of $\leq 0.7 \mathrm{mmol} / \mathrm{L}\left(\mathrm{MD}_{\text {cut-off }} 7.0\right)$ for $\mathrm{FPG}$ measurements used for the diagnosis of DM. At this glucose concentration of $7.0 \mathrm{mmol} / \mathrm{L}$, a SD $=0.35 \mathrm{mmol} / \mathrm{L}$ and a MD of $0.7 \mathrm{mmol} / \mathrm{L}$ (Eq. 5) correspond to a $\% \mathrm{CV}$ of $\leq 5 \%$. A $\% \mathrm{CV}$ of $6 \%$ would correspond to $0.84 \mathrm{mmol} / \mathrm{L}$, i.e. any value between 6.16 and 7.84. Applied to a concentration of $5.6 \mathrm{mmol} / \mathrm{L}$ the interval would be $4.93-6.27 \mathrm{mmol} / \mathrm{L}$. An analytical \%CV of this magnitude, or larger, would therefore not differentiate between diabetes and non-diabetes. This is illustrated by the intersection and overlap of the "funnels" in Fig. 1.

Current quality assurance regulations, such as the commonly practiced Westgard control rules [8] or the compulsory Guideline of the German Medical Association on Quality Assurance in Medical Laboratory Examinations (RiliBAEK) [9], usually require at least two internal quality control (IQC) samples of different concentrations per assay, applied at least once each per instrument per day. If the daily requirements of the control system are met, no further action need be taken and the results are released. It is thus assumed that results obtained between two accepted control samples meet the quality criteria [5].

Here we report our assessment of the analytical imprecision of glucose concentration measurements on a continuously operated state-of-the-art measurement system. Glucose measurements were performed on three individual instruments connected to an automatic random sample distribution system. Two 


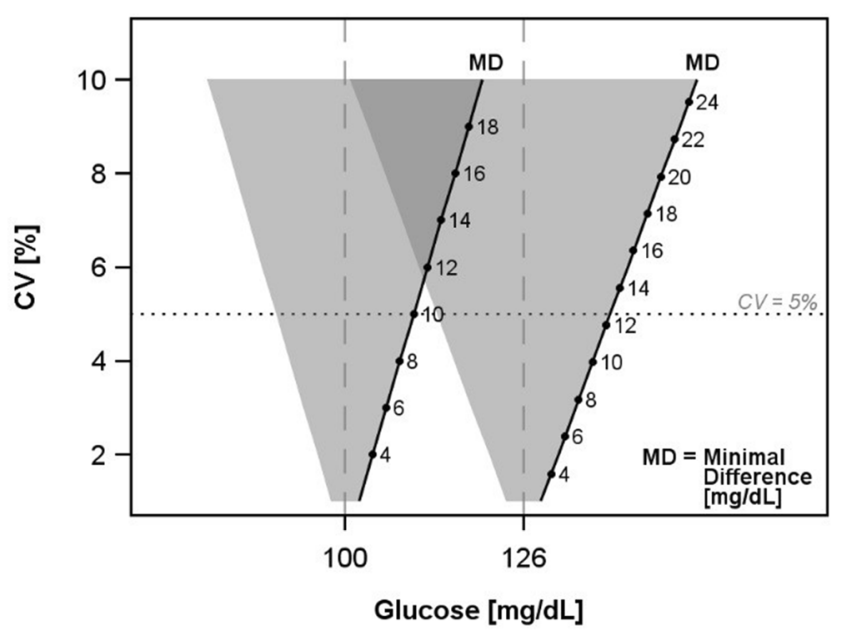

Fig. 1 Relation between coefficient of variation $(\% \mathrm{CV})$ and minimal difference $(M D)$ at glucose concentration cut-offs used for the diagnosis of diabetes mellitus. The width of the funnels at each $\% \mathrm{CV}$ is the MD and can be read on the oblique scale. The vertical dotted lines are the cut-off values. The overlapping areas above the intersection

approaches were chosen: (1) long-term imprecision calculated from IQC data obtained in a regular patient care setting between 2009 and 2018; and (2) detailed short-term imprecision assessed hourly through the measurement of additional control materials during a 1-week period.The $\mathrm{MD}_{\text {cut-off }}$ of both approaches was calculated and compared for the FPG cut-off value of $7.0 \mathrm{mmol} / \mathrm{L}(126 \mathrm{mg} / \mathrm{dL})$ for the diagnosis of DM.

The aim of the study was to provide reliable imprecision data for glucose concentration measurements and translate this imprecision into a clinical useable term, namely the MD. This information is important in view of the fact that each measurement result is subject to imprecision, and clinicians should be aware of the magnitude of this imprecision, especially when using cut-offs for diagnostic purposes, such as in the diagnosis of DM.

\section{METHODS}

All measurements were conducted at the Institute of Clinical Chemistry and Laboratory Medicine of the University Medicine of

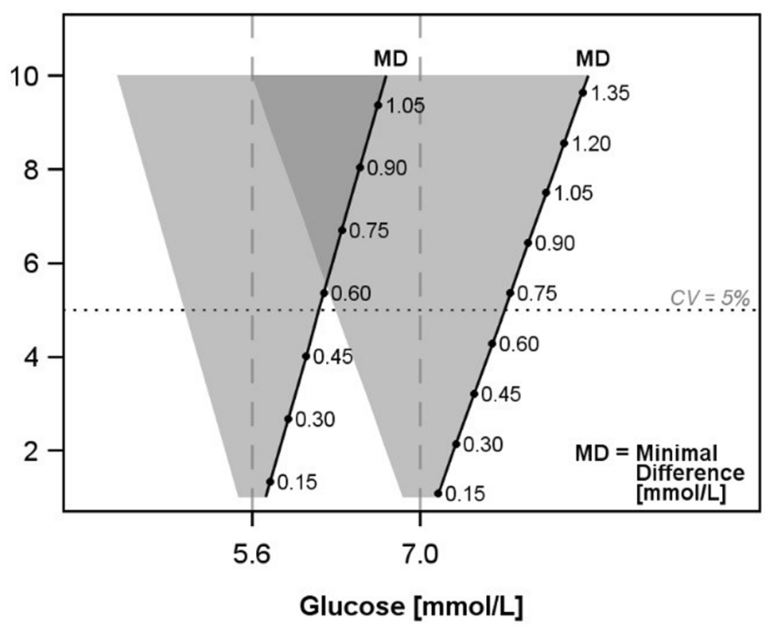

of the funnels correspond to $\% \mathrm{CVs}$ where the difference between 5.6 and $7.0 \mathrm{mmol} / \mathrm{L}$ glucose cannot be ascertained. Figure is reproduced with permission from $M$ Nauck, A Petersmann, D Müller-Wieland, et al. Definition, Klassifikation und Diagnostik des Diabetes mellitus. Diabetol Stoffwech. 2018;13

Greifswald, which operates 24 h, 7 days a week. The long-term study was performed between January 2009 and December 2018. The measuring systems were used for patient care and complied with the Rili-BAEK guideline. The plasma glucose concentrations were measured using calibrators, reagents and instruments (hexokinase-glucose-6-phosphate dehydrogenase method [10]) on three Dimension Vista 1500 (instruments 1-3) that were connected by a laboratory automation system (all from Siemens Healthcare GmbH, Eschborn, Germany). The automation system was changed from StreamLab to FlexLab (instrument 4; Siemens Healthcare $\mathrm{GmbH}$ ) in 2012. Both automation systems distributed the samples to analytical Dimension Vista instruments 1-3. The IQC material Tru Liquid Monitrol (Thermo Fisher Scientific, Schwerte, Germany) was used from 2009 until December 2013, after which time it was changed to Liquid Assayed Multiqual (BioRad Laboratories, Munich, Germany).

No patient material was used for this study; therefore, the study did not need to be registered, and it did not require the approval of an institutional ethics committee. 


\section{Minimal Difference}

The MD is based on the SD and is expressed in the unit of the measurand to enable clinicians to apply it directly to the result [7]. If a coverage factor $(k)$ of 2 is used, the MD corresponds to a confidence level of about 95\%. If two consecutive measurements are compared, the measurement uncertainty of both must be considered (Eq. 2). If measurement uncertainty is the same for each measurement, the equation can be simplified, as given in Eq. (3). If a measurement result is compared to a fixed cut-off value that is normally considered to be devoid of an uncertainty, the $\mathrm{MD}$ can be simplified to the $\mathrm{MD}_{\text {cut-off }}$ as calculated by Eq. (4), which in turn can be simplified to Eq. (5).

$$
\begin{aligned}
& \% \mathrm{CV}=\frac{\mathrm{SD}}{\bar{X}} \times 100 ; \mathrm{SD}=\frac{\bar{X} \times \% \mathrm{CV}}{100} \\
& \mathrm{MD}=k \times \sqrt{\mathrm{SD}_{1}^{2}+\mathrm{SD}_{2}^{2}} \\
& \text { If } \mathrm{SD}_{1}=\mathrm{SD}_{2}: \\
& \mathrm{MD}=k \times \sqrt{2 \times \mathrm{SD}^{2}}=2 \times \mathrm{SD} \times \sqrt{2} \\
& \text { If } \mathrm{SD}_{2}=0: \\
& \mathrm{MD}_{\text {cut }- \text { off }}=k \times \sqrt{\mathrm{SD}_{1}^{2}},
\end{aligned}
$$

which can be simplified to:

$$
\mathrm{MD}_{\text {cut-off }}=k \times \mathrm{SD}=2
$$

In Eq. (1) to (5), $\bar{X}$ is the mean and $k=2$, which represents a confidence level of $95 \%$.

\section{Long-Term Experiment}

\section{Imprecision}

Documented IQC data from the laboratory information system (LIS) were used retrospectively for the study period of 10 years. Control cycles were evaluated on a monthly basis, separately for each instrument, IQC level and much of the control material. Cycles with fewer than 15 IQC results were excluded.

\section{Short-Term Experiment}

\section{Workload}

There was a total of 2016 possible measurement time points for all four instruments. The actual number of measurements was documented in order to correctly handle interruptions for service and maintenance. The overall workload, i.e. the total number of measurements per hour of the day, was retrieved retrospectively from the LIS for each of the instruments.

\section{Imprecision}

Control material was measured hourly over a period of 1 week in September 2012. Samples were directly introduced into each Dimension Vista instrument (1-3) or randomly through FlexLab, imitating the general work flow of patient samples. The latter procedure represents instrument 4. Performance of the instruments and the measurements were independently monitored with separate IQC materials according to the rules in the Rili-BAEK. Acceptance of these rules was the inclusion criterion for the measurements of both the patient care and study samples. Short-term imprecision results were performed as if they were patient samples and collected from the LIS retrospectively; they were not reported separately, nor used in the Rili-BAEK-based IQC system. Short-term control cycles were evaluated on a daily basis from the hourly measurements, separately for each instrument, IQC level and lot number.

Three levels of control material with concentrations of about 3, 7 and $20 \mathrm{mmol} / \mathrm{L}$ (BioRad Liquid Assayed Multiqual; lot no. 45,631-1 through -3; Bio-Rad Laboratories) were used. For each level, a sufficient volume of each material to cover the needs for 1 day was thawed and pooled for each concentration and then aliquoted into barcode-labeled and capped tubes suitable for use in the instruments. The aliquoted material was stored at $2-5^{\circ} \mathrm{C}$ until use (maximum storage time $24 \mathrm{~h}$ ).

\section{Calculations and Statistics}

Calculation of the \%CV and MD, including descriptive statistics and boxplots, was 
performed using $\mathrm{R}$ (version 3.5.0; release 23 March 2018; https://www.r-project.org/). Statistical differences between instruments and years were calculated using analysis of variance, and Tukey's HSD test was used as the post hoc test. Frequency graphs were designed using SAS version 9.4 software (SAS Institute Inc., Cary, NC, USA). The MD was calculated using Eq. (5), i.e. under the assumption that the cut-off was not liable to an uncertainty. All calculations are given in Electronic Supplementary Material (ESM) S1.

In the long-term approach, the MDs were calculated monthly for each instrument using the IQC results and then cumulated as a moving average to obtain a reliable value [11]. In the short-term approach, the MDs were calculated daily for each instrument. Equations (1) and (5) were applied for both approaches. All MDs were summarized in boxplots for the whole study period and also reported in detail by years and instruments, respectively.

The 95th percentiles of the $\mathrm{MD}_{\text {cut-off }}$ distributions were used to obtain reliable values for the MD suitable for the diagnosis of DM. The $\mathrm{MD}_{\text {cut-off }}$ from the different concentrations tested in this study were used to calculate a linear equation that would allow the $\mathrm{MD}_{\text {cut-off }}$ for other concentrations, such as the $\mathrm{MD}_{\text {cut-off 7.0, }}$ to be estimated.

Since the material used in the short-term experiment was pooled and then distributed to the instruments, an evaluation of the bias among the instruments was possible. This bias was calculated separately for each instrument by subtracting the mean $\mathrm{MD}_{\text {cut-off }}$ of each instrument from the median $\mathrm{MD}_{\text {cut-off }}$ of all instruments.

Compliance with Ethics Guidelines This article does not contain any studies with human participants or animals performed by any of the authors.

\section{RESULTS}

\section{Long-Term Experiment}

\section{Imprecision}

Long-term glucose measurement imprecision was calculated from the IQC samples tested during patient care under routine conditions. The calculated values converged and reached a stable level after 30 observations. All results were obtained following the Rili-BAEK and are summarized in Fig. 2. The $\mathrm{MD}_{\text {cut-off } 7.0}$ was calculated from the linear equation $y=0.065 x-$ 0.013 , which was derived from four concentrations $(3.2,5.5,15.9$, and $19.2 \mathrm{mmol} / \mathrm{L})$ and determined to be $0.44 \mathrm{mmol} / \mathrm{L}$ (Fig. 2c). The $\mathrm{MD}_{\text {cut-off } 7.0}$ differed marginally but significantly between the instruments (ESM S2, S3). This tendency was also found for a few IQCs, which were found to be higher in 2008 than in any other year; after a change in IQCs in 2014, the performance was stable throughout all years, i.e. there was no significant difference in the $\mathrm{MD}_{\text {cut-off } 7.0}$ (ESM S2).

\section{Short-Term Experiment}

All measurements were performed together with measurements taken for patient care under routine conditions. All results were complied using Rili-BAEK. Since the regular IQC samples were not part of the study design in the shortterm approach, the \%CVs from the routine IQC were taken separately: 2.6 and $1.9 \%$ at a glucose concentration of 5.2 and $14.7 \mathrm{mmol} / \mathrm{L}$, respectively.

Evaluation of the bias among the instruments was possible since the sample material used in the short-term experiment was pooled and then distributed across the instruments. The bias of the median concentration ranged from -0.1 to $0.1 \mathrm{mmol} / \mathrm{L}$. Relative contributions of each instrument to the workload are given in Fig. 3.

\section{Workload Profiles}

A total of 1773 hourly glucose measurements for all concentrations was available for the analysis, which corresponds to $88 \%$ of all possible $(n=2016)$ measurements. The measurements that were missing were due to daily service and maintenance procedures. Examination of the daily workload profile, including all analytes provided for regular patient care on the Vista instruments (Fig. 3a), revealed one main peak at about 9-10 a.m., preceded by a smaller 

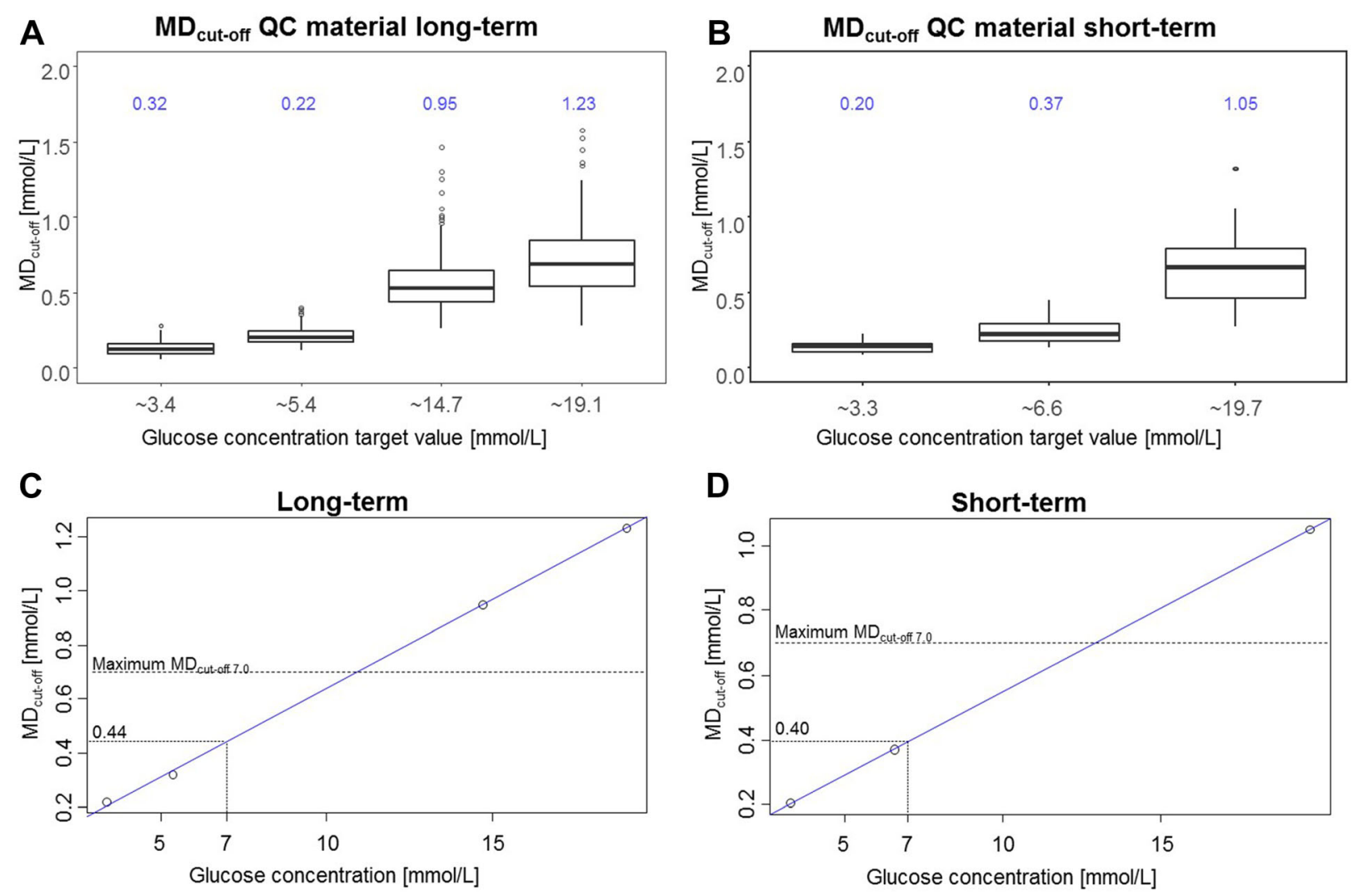

Fig. 2 Distribution of the MD cut-offs $\left(M_{\text {cut-off }}\right)$ at different concentrations of glucose $(\mathbf{a}, \mathbf{b})$ and linear regressions $(\mathbf{c}, \mathbf{d})$ based on the 95th percentile $\mathrm{MD}_{\text {cut-off }}$ (values are given above the boxplots in $\mathbf{a}$ and $\mathbf{b})$. The linear regression (blue solid line) can be used to derive the $\mathrm{MD}$ at a plasma glucose cut-off value of $7.0 \mathrm{mmol} / \mathrm{L}\left(M D_{\text {cut-off } 7.0)}\right.$

as indicated by the solid black line. The maximum $\mathrm{MD}_{\text {cut-off } 7.0}$ according to the German Diabetes Association (DDG) is marked by the horizontal dotted line. a, c Long-term internal quality control (IQC) data from 2009 to $2018, \mathbf{b}, \mathbf{d}$ short-term data from hourly measurements of QC material during 1 week
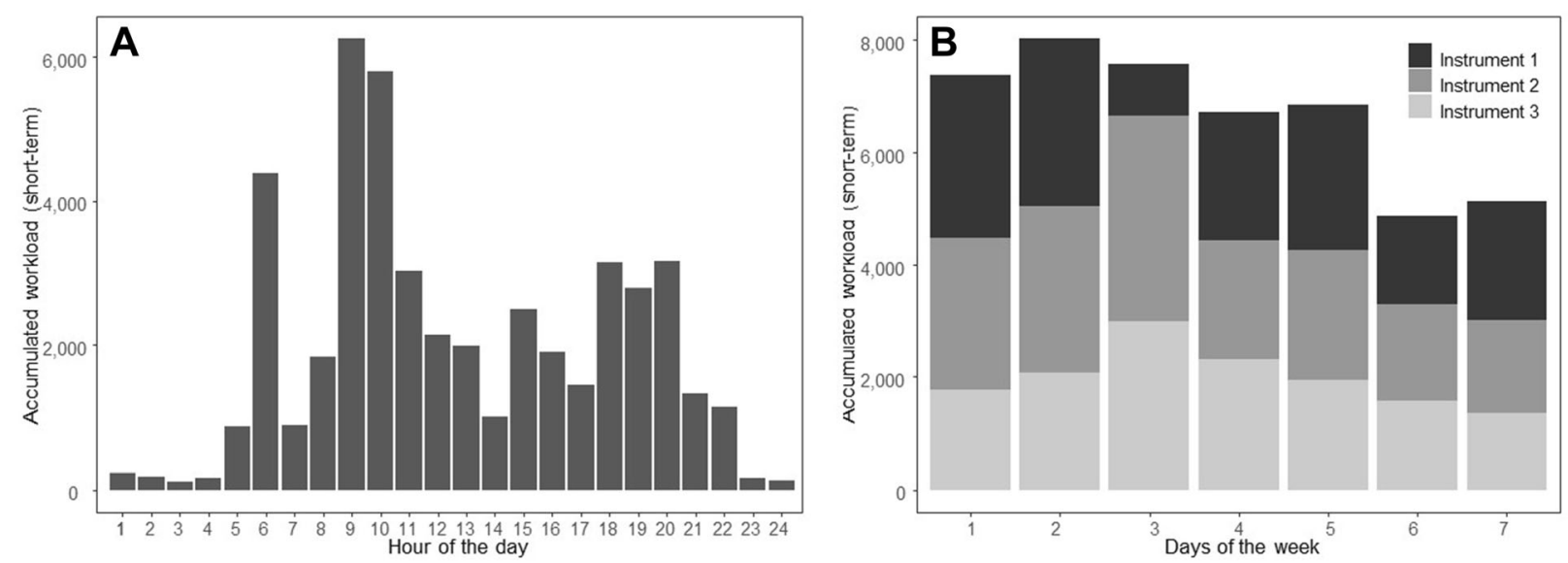

Fig. 3 Workload of all instruments in number of tests according to hour of the day (a) and day of the week (b) 
peak at 6 a.m., with a range of approximately 4000-6000 accumulated tests over the study period of 7 days. Between 500 and 800 laboratory tests were performed during these heavy workload hours. The lowest number of tests was recorded between 11 p.m and 4 a.m. The workload was shared approximately equally between the instruments, except for day 3 when two instruments took over most of the workload (Fig. 3b). Variations in results between and within days were noticeable but small (ESM S4) and showed no dependence on the workload.

\section{Imprecision}

The $\mathrm{MD}_{\text {cut-off }}$ of the three investigated glucose concentrations $(3.3,6.6$ and $19.7 \mathrm{mmol} / \mathrm{L})$ are summarized in Fig. 2. The linear regression $(y=0.052 x+0.032 ; \quad$ Fig. $2 d)$ of the three obtained $\mathrm{MD}_{\text {cut-off }}$ values enabled the calculation of the $\mathrm{MD}_{\text {cut-off } 7.0 \text {, which was } 0.40 \mathrm{mmol} /}$ L.

The design of the short-term experiment permitted a more detailed evaluation, such as reporting daily variations within instruments for the investigated concentrations. In the short-term approach there was no significant difference between the $\mathrm{MD}_{\text {cut-off } 7.0}$ of the individual instruments or between days. The $\mathrm{MD}_{\text {cut- }}$ off 7.0 of instrument 4 (Flexlab) was slightly but significantly higher than that of the other instruments. The $\mathrm{MD}_{\text {cut-off } 7.0}$ of the short-term experiment was comparable to that from the long-term experiment. The measurements results are summarized and reported in detail by day and instrument in ESM S4.

\section{DISCUSSION}

Clinicians should be aware of measurement uncertainty, such as, for example, when comparing the result of a glucose concentration measurement to a cut-off value used for the diagnosis of DM.

The $\mathrm{MD}_{\text {cut-off }}$ is a metric denoting the smallest analytical difference between a measurement and a cut-off that can be regarded as statistically significant at a 95\% confidence level-provided a coverage factor $(k)$ of 2 is applied [12]. Since the $\mathrm{MD}_{\text {cut-off }}$ is given in the unit of the analyte it can only be reported for a specific concentration. As the subject of our study was the $\mathrm{MD}_{\text {cut-off }}$ for the diagnosis of $\mathrm{DM}$ we focused on glucose concentrations at 7.0 $\mathrm{mmol} / \mathrm{L}$, which is the diagnostic cut-off value for FPG as recommended by ADA and DDG and assessed long-term and short-term imprecision expressed as $\mathrm{MD}_{\text {cut-off } 7.0}$ for glucose concentration measurements.

The $\mathrm{MD}_{\text {cut-off } 7.0}$ for glucose in the long-term and the short-term approaches were 0.44 and $0.40 \mathrm{mmol} / \mathrm{L}$, respectively. The relation between the $\mathrm{MD}_{\text {cut-off }}$ and the glucose concentration represents the relative $\mathrm{SD}$, i.e. the $\% \mathrm{CV}$. Any $\mathrm{MD}_{\text {cut-off }}$ on the linear regression line in Fig. 2 represents the identical \%CV. A MD cut-off above and below this regression line represents a higher and a lower \%CV, respectively. In the laboratory setting, \%CV can also be used to compare imprecisions of different quality control concentrations.

In our study, the $\mathrm{MD}_{\text {cut-off } 7.0}$ remained below the recommended limit of $0.7 \mathrm{mmol} / \mathrm{L}$ in both approaches. The results of the study illustrate the MD concept [6] as follows:

1. It can be expected that $5 \%$ of all glucose concentration measurements fall outside this $\mathrm{MD}_{\text {cut-off. }}$ The central $95 \%$ interval of all measurement results in the short-term study was found to be $0.4-0.5 \mathrm{mmol} / \mathrm{L}$ at a glucose concentration of $6.6 \mathrm{mmol} / \mathrm{L}$ (ESM S4). This interval should resemble the $\mathrm{MD}_{\text {cut-off } 7.0}$ derived from the long-term experiment, which in turn was shown to be $0.44 \mathrm{mmol} / \mathrm{L}$.

2. The differences between the highest and lowest glucose concentrations of study samples in the short-term experiment at $6.6 \mathrm{mmol} / \mathrm{L}$ varied between 0.6 and $0.7 \mathrm{mmol} / \mathrm{L}$. Since a coverage factor $k=2$ was chosen in this study, this result is reasonable. If the coverage factor $(k)$ is increased to 3 to correspond to a 99\% level of confidence, the $\mathrm{MD}_{\text {cut-off }}$ from the longterm study would be $0.67 \mathrm{mmol} / \mathrm{L}$, which also matches our findings for minimum and maximum results in the short-term study.

In addition, routine Rili-BAEK IQC in the short-term approach, which relied on separate 
control material, confirmed that the $\% \mathrm{CV}$ at a concentration of $6.6 \mathrm{mmol} / \mathrm{L}$ was $2.6 \%$, which corresponds to a $\mathrm{MD}_{\text {cut-off }}$ of $0.5 \mathrm{mmol} / \mathrm{L}$. This also resembles the central 95\% interval of all measurement results in the short-term study.

The results of the present study are also in line with the $\mathrm{MD}_{\text {cut-off }}$ of $0.38 \mathrm{mmol} / \mathrm{L}$ at $5.0 \mathrm{mmol} / \mathrm{L}$ calculated from approximately 21,000 duplicate measurements of glucose in plasma collected from patients instead of from IQC material [13]. This result demonstrates that the control material used gives $\mathrm{MD}_{\text {cut-off }}$ results comparable to those determined for plasma samples from patients and therefore allows the $\mathrm{MD}_{\text {cut-off }}$ to be extrapolated to glucose concentration measurements of plasma samples from patients. Imprecision performance data claimed by the manufacturer are a $\mathrm{SD}=0.12 \mathrm{mmol} / \mathrm{L}$ at $4.12 \mathrm{mmol} / \mathrm{L}$ and a $\mathrm{SD}=0.46 \mathrm{mmol} / \mathrm{L}$ at $21.02 \mathrm{mmol} / \mathrm{L}$, both of which can be used to derive a $\mathrm{MD}_{\text {cut-off } 7.0}$ of 0.36 (linear regression of $y=0.0402 x+0.0742)$ [14]. The imprecision given by the manufacturer is based on CLSI/ National Committee for Clinical Laboratory Standard(NCCLS) EP5-A2: measurement of four samples of each concentration per day in two separate runs of two samples each for 20 days [4]. These imprecision results are lower than our findings indicating that imprecision based on the CLSI/NCCLS EP5-A2 protocol is not sufficient to provide a reliable $\mathrm{MD}_{\text {cut-off }}$.

The $\mathrm{MD}_{\text {cut-off } 7.0}$ were calculated from glucose concentration results obtained from three instruments run in parallel. The $\mathrm{MD}_{\text {cut-off }}$ for individual instruments differed significantly in the long-term approach, but not in the shortterm approach, indicating that a longer period of time is needed for an assessment of a reliable $\mathrm{MD}_{\text {cut-off } 7.0}$. Furthermore, the $\mathrm{MD}_{\text {cut-off }}$ for the individual instruments were slightly lower than those from the overall system; this result was expected since in addition to the larger imprecision, slight biases between the instruments also occurred, thereby adding to the variation when examining the overall distribution system 'instrument 4.'

The results reported to clinicians, however, represent the combined performance of all instruments. The results of this study show that even when each instrument is shown to have a good assay performance, the clinician would experience differences in glucose results as large as $1.0 \mathrm{mmol} / \mathrm{L}$ at a glucose concentration of $6.6 \mathrm{mmol} / \mathrm{L}$ (minimum $6.2 \mathrm{mmol} / \mathrm{L}$; maximum $7.2 \mathrm{mmol} / \mathrm{L}$ ) due to imprecision and bias among the instruments. Therefore, the $\mathrm{MD}_{\text {cut-off }}$ calculation should be based on MD distribution from all instruments that are connected to the automated distribution system.

Data from the long-term study show a reliable stability of the investigated systems over one decade, demonstrating the high quality of the manufacturer and medical laboratories. The effectiveness of the quality assurance systems, such as Rili-BAEK, with internal and external quality controls also contribute to the stable performance over time.

Short-term data showed minor performance shifts within the instruments, but these could not be linked to shifts in the workload. Even though the systems were observed to have a high stability, it has to be noted that the minimum QC frequency by Rili-BAEK allowed hundreds of patient results to be released inbetween IQCs. An increase IQC frequency would reduce the number of released patient results, but it cannot be excluded that incidences between two IQC results may still occur unnoticed. Still, a higher IQC frequency reduces the number of samples that need to be retested after a failed IQC. It is up to the individual medical laboratory to carefully balance costs and quality of patient care.

The variability introduced by slight differences in performance within and between instruments can be covered by reporting the long-term $\mathrm{MD}_{\text {cut-off }}$ across all connected instruments. Stable results for $\mathrm{MD}_{\text {cut-off } 7.0}$ were obtained after about 30 independent control cycles of $\mathrm{MD}_{\text {cut-off } 7.0 \text {, a number that was }}$ reached after 1 year of combining $\mathrm{MD}_{\text {cut-off } 7.0}$ data from all three instruments.

The between-year $\mathrm{MD}_{\text {cut-off } 7.0}$ values were not significantly different, except for the first year of the long-term approach when the instruments had just been introduced to the laboratory. Thus, medical laboratories may use the IQC of about 1 year to provide a reliable $\mathrm{MD}_{\text {cut-off }}$ to report along with glucose concentration results used to diagnose DM. This 
finding becomes especially important when close diagnostic cut-offs increase the need for reliable results to classify individuals correctly [15]. Subsequently, continuous monitoring of the $\mathrm{MD}_{\text {cut- }}$ off builds an even more reliable database and can also facilitate identification of performance changes as well as comparisons of different measurement methods and laboratories.

\section{Limitations}

The study was limited to one type of instrument (Dimension Vista 1500), and the short-term imprecision part was limited to 1 week only. Adverse effects may have occurred less often than weekly and, therefore, these could not be identified in the short-term approach study due to its design. The commercially available IQC material used in the study closely resembles patient material, but is not patient material; therefore, effects due to any differences cannot be completely excluded. The aim of the study design was to assess measurement imprecision and express this imprecision as the MD.

\section{CONCLUSION}

Imprecision for glucose concentration measurements, assessed as $\mathrm{MD}_{\text {cut-off } 7.0}$ from monthly IQC control cycles over a period of 10 years, was $0.44 \mathrm{mmol} / \mathrm{L}$ and, therefore, well below the recommended limit of $0.7 \mathrm{mmol} / \mathrm{L}$. Hourly measurements made over a 1-week period confirmed these findings and illustrated the MD concept. Imprecision as measured by IQC is also remarkably stable over many years of operation.

Current imprecision assessment focuses only on single instruments, whereas clinicians perceive the combined analytical performance of all instruments used for a certain assay in a given laboratory. Therefore, we suggest deriving the $\mathrm{MD}_{\text {cut-off }}$ from all instruments and control cycles that are used in the setting of patient care in a given medical laboratory. In our study, about 30 independent control cycles provided sufficient data to determine a reliable $\mathrm{MD}_{\text {cut-off- Establish- }}$ ing a continuous monitoring of $\mathrm{MD}_{\text {cut-off }}$ may complement traditional quality assurance.

\section{ACKNOWLEDGEMENTS}

Funding. The study was in part funded by Bio-Rad. The Rapid Service Fee was paid for by the authors.

Medical Writing, Editorial, and other Assistance. The authors thank Stefan Pleus, Guido Freckmann and Erwin Schleicher for intense discussion of the findings and their helpful corrections of the manuscript.

Authorship. All named authors meet the International Committee of Medical Journal Editors (ICMJE) criteria for authorship for this article, take responsibility for the integrity of the work as a whole, and have given their approval for this version to be published.

Authorship Contributions. SK and AP drafted the manuscript; SZ, MD, AK and NF performed the data analyses; SK, AP, SZ, NF, AK and $\mathrm{MN}$ interpreted the data; NF, RL, LH, AK, MN revised the manuscript content. All authors read and approved the final manuscript. AP takes full responsibility for the work as a whole, including access to data and the decision to submit and publish the manuscript.

Disclosures. Sandra Keutmann, Stephanie Zylla, Mathilde Dahl, Nele Friedrich, Rüdiger Landgraf, Lutz Heinemann, Anders Kallner, Matthias Nauck and Astrid Petersmann have nothing to disclose. Astrid Petersmann is currently affiliated with the Institute of Clinical Chemistry and Laboratory Medicine, University Medicine Goettingen, Goettingen, Germany. Sandra Keutmann is currently affiliated with the Department of Anaesthesia, Trinity Hospital, Wesseling, Germany.

Compliance with Ethics Guidelines. This article does not contain any studies with human participants or animals performed by any of the authors.

Data Availability. The datasets generated and analyzed during the current study are 
available from the corresponding author on reasonable request.

Open Access. This article is licensed under a Creative Commons Attribution-NonCommercial 4.0 International License, which permits any non-commercial use, sharing, adaptation, distribution and reproduction in any medium or format, as long as you give appropriate credit to the original author(s) and the source, provide a link to the Creative Commons licence, and indicate if changes were made. The images or other third party material in this article are included in the article's Creative Commons licence, unless indicated otherwise in a credit line to the material. If material is not included in the article's Creative Commons licence and your intended use is not permitted by statutory regulation or exceeds the permitted use, you will need to obtain permission directly from the copyright holder. To view a copy of this licence, visit http://creativecommons.org/licenses/by/4. $0 /$.

\section{REFERENCES}

1. Marathe PH, Gao HX, Close KL. American Diabetes Association standards of medical care in diabetes 2017. J Diabetes. 2017;9:320-4.

2. Müller-Wieland D, Petersmann A, Nauck M, et al. Definition, classification and diagnosis of diabetes mellitus. Diabetol Stoffwechs. 2016;11:S78-81.

3. World Health Organization. Use of glycated haemoglobin (HbA1c) in the diagnosis of diabetes mellitus: abbreviated report of a WHO consultation. WHO guidelines approved by the Guidelines Review Committee. Geneva: World Health Organization; 2011.

4. Joint Committee for Guides in Metrology (JCGM). Evaluation of measurement data-guide to the expression of uncertainty in measurement. JCGM 100:2008. https://www.bipm.org/en/publications/ guides/gum.html.

5. Büttner J, Broth R, Broughton PM, Bowyer RC. International Federation of Clinical Chemistry.
Committee on standards. Expert panel on nomenclature and principles of quality control in clinical chemistry. IFCC document stage 2, draft 3; 1979-07 with a view to an IFCC recommendation. Quality control in clinical chemistry. Part 4. Internal quality control. J Clin Chem Clin Biochem 1980;18: 535-541.

6. Clinical and Laboratory Standards Institute (CLSI). Expression of measurement uncertainty in laboratory medicine; approved guideline. 2012:Document EP29.

7. Parvin CA. New insight into the comparative power of quality-control rules that use control observations within a single analytical run. Clin Chem. 1993;39:440-7.

8. Westgard JO, Barry PL, Hunt MR, Groth T. A multirule Shewhart chart for quality control in clinical chemistry. Clin Chem. 1981;27:493-501.

9. Revision of the "Guideline of the German Medical Association on quality assurance in medical lab examinations-Rili-BAEK" (unauthorized translation). J Laboratory Med. 2015;39(1):26-69.

10. Kunst A, Drager B, Ziegenhorn J. UV methods with hexokinase and glucose-6-phosphate dehydrogenase. In: Bergemeier $\mathrm{H}$, editor. Methods of enzymatic analysis. Deerfield: Verlag Chemie; 1983. p. $163-72$.

11. Badrick $T$, Cervinski $M$, Loh TP. A primer on patient-based quality control techniques. Clin Biochem. 2019;64:1-5.

12. Petersen PH, Ricos C, Stockl D, et al. Proposed guidelines for the internal quality control of analytical results in the medical laboratory. Eur J Clin Chem Clin Biochem. 1996;34:983-99.

13. Petersmann A, Wasner C, Nauck M, Kallner A. Frequency of extreme differences and clinical performance of glucose concentration measurements judged from 21,000 duplicate measurements. Clin Chem. 2013;59:998-1000.

14. Siemens Healthcare Diagnostics. Dimension Vista System reagent cartridge, glucose. Ref. K1039. Newark: Siemens Healthcare Diagnostics; 2017.

15. D'Orazio P, Burnett RW, Fogh-Andersen N, et al. Approved IFCC recommendation on reporting results for blood glucose (abbreviated). Clin Chem. 2005;51:1573-6. 